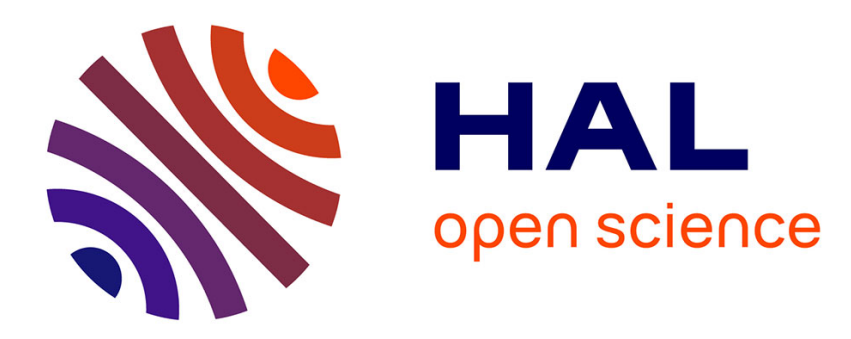

\title{
Monge-Kantorovich equation for degenerate Finsler metrics
}

\author{
Van Thanh Nguyen
}

\section{To cite this version:}

Van Thanh Nguyen. Monge-Kantorovich equation for degenerate Finsler metrics. Nonlinear Analysis:

Theory, Methods and Applications, In press. hal-03099152

\section{HAL Id: hal-03099152 \\ https://hal.science/hal-03099152}

Submitted on 6 Jan 2021

HAL is a multi-disciplinary open access archive for the deposit and dissemination of scientific research documents, whether they are published or not. The documents may come from teaching and research institutions in France or abroad, or from public or private research centers.
L'archive ouverte pluridisciplinaire HAL, est destinée au dépôt et à la diffusion de documents scientifiques de niveau recherche, publiés ou non, émanant des établissements d'enseignement et de recherche français ou étrangers, des laboratoires publics ou privés. 


\title{
Monge-Kantorovich equation for degenerate Finsler metrics
}

\author{
Van Thanh Nguyen* \\ Department of Mathematics and Statistics, Quy Nhon University, Vietnam
}

October 21, 2020

\begin{abstract}
The paper establishes that a generalization of Monge-Kantorovich equation gives rise to a necessary and sufficient optimality condition for the Kantorovich dual problem and minimal flow problem associated with a very degenerate Finsler metric without any assumption on coerciveness.
\end{abstract}

Keywords. Monge-Kantorovich equation, degenerate Finsler metric, Kantorovich dual problem, minimal flow

AMS subject classifications. 49J20, 49K20, 49Q22, 35Q93.

\section{Introduction}

Monge-Kantorovich equation appears as an optimality condition to the two following variational problems, called Kantorovich dual problem (see e.g. [13, 14]),

$$
\max _{u \in W^{1, \infty}(\Omega)}\left\{\int_{\bar{\Omega}} u \mathrm{~d}\left(f_{1}-f_{0}\right):|\nabla u| \leq 1\right\}
$$

and, called minimal flow problem (or, Beckmann's continuous model of transportation [1], see also [12]),

$$
\min _{\Phi \in \mathcal{M}(\bar{\Omega})^{N}}\left\{\int_{\bar{\Omega}} \mathrm{d}|\Phi|:-\operatorname{div} \Phi=f_{1}-f_{0} \text { in } \mathcal{D}^{\prime}(\bar{\Omega})\right\} .
$$

Here, $\Omega$ is a bounded Lispchitz domain of $\mathbb{R}^{N}$. Two finite Radon measures $f_{0}$ and $f_{1}$ on $\bar{\Omega}$ satisfy the mass balance $\int_{\bar{\Omega}} \mathrm{d} f_{0}=\int_{\bar{\Omega}} \mathrm{d} f_{1}$. The space of vector-valued finite Radon measures on $\bar{\Omega}$ is denoted by $\mathcal{M}(\bar{\Omega})^{N}$. The divergence constraint $-\operatorname{div} \Phi=f_{1}-f_{0}$ in $\mathcal{D}^{\prime}(\bar{\Omega})$

\footnotetext{
*Corresponding author. Email: nguyenvanthanh@qnu.edu.vn
} 
is understood in the sense of distribution, coupled with a homogeneous Neumann boundary condition, that is,

$$
\int_{\bar{\Omega}} \frac{\Phi}{|\Phi|} \cdot \nabla \xi \mathrm{d}|\Phi|=\int_{\bar{\Omega}} \xi \mathrm{d}\left(f_{1}-f_{0}\right), \quad \forall \xi \in C^{1}(\bar{\Omega})
$$

The Monge-Kantorovich equation has first been studied by Evans and Gangbo [7] for regular functions $f_{0}$ and $f_{1}$ with the aim of deriving the existence of optimal map to Monge's transportation problem via very deep PDE techniques. The equation was generalized to finite Radon measures $f_{0}$ and $f_{1}$ by Bouchitté and Buttazzo [5] as follows

$$
\begin{cases}-\operatorname{div}\left(\mu D_{\mu} u\right)=f_{1}-f_{0} & \text { in } \mathcal{D}^{\prime}(\bar{\Omega}) \\ \left|D_{\mu} u\right|=1 & \mu \text {-a.e. } \\ |\nabla u| \leq 1 & \text { a.e. in } \Omega\end{cases}
$$

where $D_{\mu} u$ is the tangential gradient with respect to (w.r.t.) the measure $\mu$. It turns out to be the standard gradient $\nabla u$ if $\mu$ is absolutely continuous w.r.t. the Lebesgue measure.

It is well known that $\phi:=\mu D_{\mu} u$ is an optimal flow to the minimal flow problem (1.1), which is closely related to the Monge-Kantorovich transport problem with the Euclidean distance cost.

These relations were also generalized to Finsler metric costs in (1.1) of the form

$$
\int_{\bar{\Omega}} F\left(x, \frac{\Phi}{|\Phi|}\right) \mathrm{d}|\Phi| .
$$

Let $\omega:=\{x \in \bar{\Omega}: F(x,.) \equiv 0\}$. Finsler metric $F$ is called coercive on $\bar{\Omega} \backslash \omega$ if there exist positive constants $c_{1}, c_{2}$ such that $c_{1}|p| \leq F(x, p) \leq c_{2}|p| \forall x \in \bar{\Omega} \backslash \omega$. Monge-Kantorovich equation for such a coercive Finsler metric is studied in [3] under the assumption $\omega$ being a regular domain. Related works for non-degenerate Finsler metrics (i.e., coercive on the whole $\bar{\Omega}$ and $\omega=\emptyset)$ are also discussed in $[2,8,10]$. The case of $F(x, p) \equiv F_{1}(p)$ (independent of $x$ ) is treated in [11], where $F_{1}($.$) is a continuous even convex function$ satisfying $F_{1}(p) \rightarrow+\infty$ as $p \rightarrow+\infty$.

The purpose of this paper is to derive the Monge-Kantorovich equation as a necessary and sufficient optimality condition for degenerate Finsler metrics without any assumption on coerciveness. This generalization is useful in view of applications such as, for example, $F(x, p)=k(x)|p|$ for a nonnegative continuous function $k$. It is clear that $F$ is not necessarily coercive on the set $\bar{\Omega} \backslash \omega$ with $\omega:=\{x \in \bar{\Omega}: k(x)=0\}$. To obtain the result in general case, the main difficulty corresponds to finding a sequence of smooth approximation functions satisfying a similar property of 1-Lipschitz functions w.r.t. such a degenerate Finsler metric. 
The paper is organized as follows. We present some preliminaries in Section 2. Section 3 is devoted to the smooth approximation issue. Finally, the Monge-Kantorovich equation for degenerate Finsler metrics is presented and proven in Section 4.

\section{Preliminaries}

\subsection{Degenerate Finsler metrics}

Let $\Omega$ be a bounded Lipschitz domain of $\mathbb{R}^{N}$. A Finsler metric is a continuous function $F: \bar{\Omega} \times \mathbb{R}^{N} \longrightarrow[0,+\infty)$ such that $F(x,$.$) is convex and positively 1-homogeneous w.r.t.$ the second variable, i.e., $F(x, t p)=t F(x, p) \forall(x, p) \in \bar{\Omega} \times \mathbb{R}^{N}$ and $t \geq 0$. Its polar function $F^{*}$ is defined by

$$
F^{*}(x, q)=\sup _{\{p: F(x, p) \leq 1\}}\langle q, p\rangle .
$$

As a typical example, $F(x, p)=k(x)|p|$ and $F^{*}(x, q)=\frac{1}{k(x)}|q|$ for any nonnegative continuous function $k$ on $\bar{\Omega}$, with the convention $\frac{0}{0}=0$. Another example in $1 \mathrm{D}$ is $F(x, p)=k_{1}(x) p^{-}+k_{2}(x) p^{+}$and $F^{*}(x, q)=\frac{1}{k_{1}(x)} q^{-}+\frac{1}{k_{2}(x)} q^{+}$for two nonnegative continuous functions $k_{1}$ and $k_{2}$, where $p^{+}=\max (p, 0), p^{-}=\max (-p, 0)$.

Let $\omega:=\{x \in \bar{\Omega}: F(x,.) \equiv 0\}$ be a closed set. As in [3], we assume that $\omega$ is a regular domain, but without any assumption about coerciveness on $\bar{\Omega} \backslash \omega$. More precisely, in this paper, we make use of the following assumption:

(A): $\quad \omega \Subset \Omega$ is a Lipschitz domain and $F(x, p) \neq 0$ for any $x \in \bar{\Omega} \backslash \omega, \quad 0 \neq p \in \mathbb{R}^{N}$.

\subsection{Duality issue}

Given two finite Radon measures $f_{0}$ and $f_{1}$ on $\bar{\Omega}$ satisfying $\int_{\bar{\Omega}} \mathrm{d} f_{0}=\int_{\bar{\Omega}} \mathrm{d} f_{1}$. We consider the Beckmann problem for a degenerate Finsler metric $F$ as

$$
\min _{\Phi \in \mathcal{M}(\bar{\Omega})^{N}}\left\{\int_{\bar{\Omega}} F\left(x, \frac{\Phi}{|\Phi|}(x)\right) \mathrm{d}|\Phi|(x):-\operatorname{div} \Phi=f_{1}-f_{0} \text { in } \mathcal{D}^{\prime}(\bar{\Omega})\right\} .
$$

In the case of non-degenerate Finsler metric $F$ (i.e., there exist $c_{1}>0, c_{2}>0$ such that $\left.c_{1}|p| \leq F(x, p) \leq c_{2}|p| \forall(x, p) \in \bar{\Omega} \times \mathbb{R}^{N}\right)$, the existence of optimal flow $\Phi$ to $\min (B)$ can be guaranteed by the direct method. When $F$ is degenerate, the existence can be obtained via optimal transport and the disintegration theorem.

The dual problem of (B) is given by

$$
\max _{u \in W^{1, \infty}(\Omega)}\left\{\int_{\bar{\Omega}} u \mathrm{~d}\left(f_{1}-f_{0}\right): F^{*}(x, \nabla u(x)) \leq 1 \text { a.e. in } \Omega\right\} .
$$


The existence of optimal solutions to $\max (D P)$ can be shown by the direct method.

Proposition 2.1. We have the duality relation

$$
\begin{aligned}
& \min _{\Phi \in \mathcal{M}(\bar{\Omega})^{N}}\left\{\int_{\bar{\Omega}} F\left(x, \frac{\Phi}{|\Phi|}(x)\right) \mathrm{d}|\Phi|(x):-\operatorname{div} \Phi=f_{1}-f_{0} \text { in } \mathcal{D}^{\prime}(\bar{\Omega})\right\} \\
& =\max _{u \in W^{1, \infty}(\Omega)}\left\{\int_{\bar{\Omega}} u \mathrm{~d}\left(f_{1}-f_{0}\right): F^{*}(x, \nabla u(x)) \leq 1 \text { a.e. in } \Omega\right\} .
\end{aligned}
$$

Proof. As usual, it is not difficult to show that $\max (D P) \leq \min (B)$ by taking $u$ as a test function in the divergence constraint. It remains to prove the inverse inequality. Consider the functional $\mathcal{H}: L^{\infty}(\Omega)^{N} \longrightarrow(-\infty,+\infty]$ defined by

$$
\mathcal{H}(z)=\inf _{u \in W^{1, \infty}(\Omega)}\left\{-\int_{\bar{\Omega}} u \mathrm{~d}\left(f_{1}-f_{0}\right): F^{*}(x, \nabla u(x)+z(x)) \leq 1 \text { a.e. in } \Omega\right\} .
$$

Then we can check at once that $\mathcal{H}$ is convex and lower semicontinuous in $L^{\infty}(\Omega)^{N}$, equipped with the weak* topology. And therefore $\mathcal{H}(0)=\mathcal{H}^{* *}(0)$.

For any $\Phi \in L^{1}(\Omega)^{N}$, we have

$$
\mathcal{H}^{*}(\Phi)=\sup _{z \in L^{\infty}(\Omega)^{N}}\langle\Phi, z\rangle-\mathcal{H}(z)=\sup _{\substack{z \in L^{\infty}(\Omega)^{N} \\ u \in W^{1, \infty}(\Omega) \\ F^{*}(x, \nabla u(x)+z(x)) \leq 1}}\langle\Phi, z\rangle+\int_{\bar{\Omega}} u \mathrm{~d}\left(f_{1}-f_{0}\right) .
$$

Set $v=\nabla u+z$, and $z=v-\nabla u$, we get

$$
\begin{aligned}
\mathcal{H}^{*}(\Phi)= & \sup _{\substack{v \in L^{\infty}(\Omega)^{N} \\
F^{*}(x, v(x)) \leq 1}}\langle\Phi, v\rangle+\sup _{u \in W^{1, \infty}(\Omega)}\langle\Phi,-\nabla u\rangle+\int_{\bar{\Omega}} u \mathrm{~d}\left(f_{1}-f_{0}\right) \\
= & \int_{\Omega} F(x, \Phi(x)) \mathrm{d} x+\sup _{u \in W^{1, \infty}(\Omega)}\langle\Phi,-\nabla u\rangle+\int_{\bar{\Omega}} u \mathrm{~d}\left(f_{1}-f_{0}\right) .
\end{aligned}
$$

Observe that if $\mathcal{H}^{*}(\Phi)<+\infty$, we obtain

$$
\langle\Phi, \nabla u\rangle=\int_{\bar{\Omega}} u \mathrm{~d}\left(f_{1}-f_{0}\right) \forall u \in W^{1, \infty}(\Omega) .
$$

In other words, $-\operatorname{div} \Phi=f_{1}-f_{0}$ in $\mathcal{D}^{\prime}(\bar{\Omega})$. Moreover,

$$
\begin{aligned}
\mathcal{H}^{* *}(0) & =\sup _{\Phi \in L^{1}(\Omega)^{N}}-\mathcal{H}^{*}(\Phi) \\
& =-\inf _{\Phi \in L^{1}(\Omega)^{N}}\left\{\int_{\Omega} F(x, \Phi(x)) \mathrm{d} x:-\operatorname{div} \Phi=f_{1}-f_{0} \text { in } \mathcal{D}^{\prime}(\bar{\Omega})\right\} .
\end{aligned}
$$

Finally, $\max (D P)=-\mathcal{H}(0)=-\mathcal{H}^{* *}(0) \geq \min (B)$, which completes the proof. 


\section{Approximation by smooth functions}

Lemma 3.1. Let $F$ be a degenerate Finsler metric satisfying the assumption (A) and $u$ be a Lipschitz function such that $F^{*}(x, \nabla u(x)) \leq 1$ a.e. in $\Omega$.

(i) Then there exists a sequence of smooth functions $u_{\varepsilon} \in C_{c}^{\infty}\left(\mathbb{R}^{N}\right)$ such that $u_{\varepsilon} \rightrightarrows u$ uniformly on $\bar{\Omega}$ as $\varepsilon \rightarrow 0$ and $\limsup _{\varepsilon \rightarrow 0} F^{*}\left(x, \nabla u_{\varepsilon}(x)\right) \leq 1$ for all $x \in \bar{\Omega}$.

(ii) In addition, if $F^{*}(x, q)$ is bounded from above on $(\bar{\Omega} \backslash \omega) \times\{|q| \leq 1\}$ then there exists a sequence of smooth functions $u_{\varepsilon} \in C_{c}^{\infty}\left(\mathbb{R}^{N}\right)$ such that $u_{\varepsilon} \rightrightarrows u$ uniformly on $\bar{\Omega}$ as $\varepsilon \rightarrow 0$ and $F^{*}\left(x, \nabla u_{\varepsilon}(x)\right) \leq 1$ for all $x \in \bar{\Omega}$ without passing to the limit. In particular, this holds whenever $F$ is a non-degenerate Finsler metric.

Remark 3.2. This lemma generalizes [8, Lemma 3.2], [9, Lemma A.1].

Before giving the proof, let us comment that the main difficulty comes from the approximation near to the boundary as well as the degeneracy of $F$.

Proof. Let $\widetilde{u}$ be the extension of $u$ on $\mathbb{R}^{N}$ by the value zero outside $\bar{\Omega}$, i.e.,

$$
\widetilde{u}(x):= \begin{cases}u(x) & \text { if } x \in \bar{\Omega} \\ 0 & \text { if } x \in \mathbb{R}^{N} \backslash \bar{\Omega} .\end{cases}
$$

Step 1 (Approximating at points near the boundary of $\omega$ ). Fix any $z \in \partial \omega$. Since $\omega$ is a Lipschitz domain, there exist $r_{z}>0$ and a Lipschitz continuous function $\gamma_{z}: \mathbb{R}^{N-1} \longrightarrow \mathbb{R}$ such that (up to rotating and relabeling if necessary)

$$
\omega \cap B\left(z, r_{z}\right)=\left\{x \mid x_{N}>\gamma_{z}\left(x_{1}, \ldots, x_{N-1}\right)\right\} \cap B\left(z, r_{z}\right) .
$$

In other words, the boundary of $\omega$ is locally expressed as the graph of a Lipschitz continuous function in $(N-1)$-dimension. Set $U_{z}:=\omega \cap B\left(z, \frac{r_{z}}{2}\right)$. Define a translation $T_{z}^{\varepsilon}: \mathbb{R}^{N} \longrightarrow \mathbb{R}^{N}$ given by $T_{z}^{\varepsilon}(x)=x+\varepsilon \lambda_{z} e_{N}$, where we choose a fixed sufficiently large $\lambda_{z}$ and all small $\varepsilon$, namely fixed $\lambda_{z} \geq \operatorname{Lip}\left(\gamma_{z}\right)+1,0<\varepsilon<\frac{r_{z}}{2\left(\lambda_{z}+1\right)}, e_{N}$ is the $N^{t h}$ element of canonical basis in $\mathbb{R}^{N}$. By this choice and the Lipschitz property of $\gamma_{z}$, we see that

$$
B\left(T_{z}^{\varepsilon}(x), \varepsilon\right) \subset \omega \cap B\left(z, r_{z}\right) \text { for all } x \in U_{z} .
$$

Let us define

$$
\widetilde{u}_{\varepsilon}(x):=\int_{\mathbb{R}^{N}} \rho_{\varepsilon}(y) \widetilde{u}\left(T_{z}^{\varepsilon}(x)-y\right) \mathrm{d} y=\int_{B\left(T_{z}^{\varepsilon}(x), \varepsilon\right)} \rho_{\varepsilon}\left(T_{z}^{\varepsilon}(x)-y\right) \widetilde{u}(y) \mathrm{d} y \text { for all } x \in \mathbb{R}^{N},
$$

where $\rho_{\varepsilon}$ is the standard mollifier on $\mathbb{R}^{N}$. It is clear that $\widetilde{u}_{\varepsilon} \in C_{c}^{\infty}\left(\mathbb{R}^{N}\right)$. Using $(3.2),(3.3)$ and the continuity of $u$ on $\bar{\Omega}$, we get

$$
\widetilde{u}_{\varepsilon} \rightrightarrows u \text { on } \bar{U}_{z} .
$$


Now, by the compactness of $\partial \omega$ and $\partial \omega \subset \bigcup_{z \in \partial \omega} B\left(z, \frac{r_{z}}{2}\right)$, there exist finite points $z_{1}, \ldots, z_{m} \in$ $\partial \omega$ such that

$$
\partial \omega \subset \bigcup_{i=1}^{m} B\left(z_{i}, \frac{r_{z_{i}}}{2}\right)
$$

By similar arguments, there exist also finite points $z_{m+1}, \ldots, z_{n} \in \partial \Omega$ such that

$$
\partial \Omega \subset \bigcup_{i=m+1}^{n} B\left(z_{i}, \frac{r_{z_{i}}}{2}\right)
$$

and (3.2), (3.3) and (3.4) hold at $z=z_{i}, i=1, \ldots, n$.

Step 2 (Approximating at points far from the boundaries). Let $U_{0} \Subset \operatorname{int}(\omega) \cup$ $(\Omega \backslash \omega)$ be an open subset such that

$$
\bar{\Omega} \subset \bigcup_{i=1}^{n} B\left(z_{i}, \frac{r_{z_{i}}}{2}\right) \bigcup U_{0}
$$

Let $\{\phi\}_{i=0}^{n}$ be a smooth partition of unity on $\bar{\Omega}$, subordinate to $\left\{U_{0}, B\left(z_{1}, \frac{r_{z_{1}}}{2}\right), \ldots, B\left(z_{n}, \frac{r_{z_{n}}}{2}\right)\right\}$ (see e.g. [6, Chapter 9]), that is,

$$
\left\{\begin{array}{l}
\phi_{i} \in C_{c}^{\infty}\left(\mathbb{R}^{N}\right), 0 \leq \phi_{i} \leq 1 \forall i=0, \ldots, n \\
\operatorname{supp}\left(\phi_{i}\right) \Subset B\left(z_{i}, \frac{r_{z_{i}}}{2}\right) \forall i=1, \ldots, n, \operatorname{supp}\left(\phi_{0}\right) \Subset U_{0} \\
\sum_{i=0}^{n} \phi_{i}(x)=1 \text { for all } x \in \bar{\Omega} .
\end{array}\right.
$$

For short, we will write $T_{i}^{\varepsilon}, U_{i}$ from Step 1 instead of $T_{z_{i}}^{\varepsilon}, U_{z_{i}}$ and $x_{i}^{\varepsilon}=x_{z_{i}}^{\varepsilon}=T_{i}^{\varepsilon}(x), i=$ $1, \ldots, n$.

Due to Step 1 , there exist $\widetilde{u}_{\varepsilon}^{1}, \ldots, \widetilde{u}_{\varepsilon}^{n} \in C_{c}^{\infty}\left(\mathbb{R}^{N}\right)$ such that

$$
\widetilde{u}_{\varepsilon}^{i} \rightrightarrows u \text { on } \bar{U}_{i}, i=1, \ldots, n .
$$

For $i=0$, since $U_{0} \Subset \operatorname{int}(\omega) \cup(\Omega \backslash \omega)$, we can define $T_{0}^{\varepsilon}(x) \equiv x$ and the standard convolution $\widetilde{u}_{\varepsilon}^{0}:=\rho_{\varepsilon} \star \widetilde{u}$. Then $\widetilde{u}_{\varepsilon}^{0} \in C_{c}^{\infty}\left(\mathbb{R}^{N}\right)$ and $\widetilde{u}_{\varepsilon}^{0} \rightrightarrows u$ on $\bar{U}_{0}$.

Step 3 (proof of (i)). Set

$$
u_{\varepsilon}(x):=\sum_{i=0}^{n} \phi_{i}(x) \widetilde{u}_{\varepsilon}^{i}(x)=\sum_{i=0}^{n} \phi_{i}(x) \int_{B\left(T_{i}^{\varepsilon}(x), \varepsilon\right)} \rho_{\varepsilon}\left(T_{i}^{\varepsilon}(x)-y\right) \widetilde{u}(y) \mathrm{d} y \text { for all } x \in \mathbb{R}^{N}
$$


Let us show that the sequence $\left\{u_{\varepsilon}\right\}$ satisfies the desired properties. We check at once that $u_{\varepsilon} \in C_{c}^{\infty}\left(\mathbb{R}^{N}\right)$ and

$$
u_{\varepsilon} \rightrightarrows \sum_{i=0}^{n} \phi_{i} u=u \text { on } \bar{\Omega} .
$$

It remains to prove that $\limsup _{\varepsilon \rightarrow 0} F^{*}\left(x, \nabla u_{\varepsilon}(x)\right) \leq 1$ for all $x \in \bar{\Omega}$. By the construction, $B\left(x_{i}^{\varepsilon}, \varepsilon\right) \subset \Omega$ for sufficiently small $\varepsilon$ and $x_{i}^{\varepsilon} \rightarrow x$ as $\varepsilon \rightarrow 0$. Observe that

$$
\begin{aligned}
\nabla u_{\varepsilon}(x) & =\sum_{i=0}^{n} \nabla \phi_{i}(x) \widetilde{u}_{\varepsilon}^{i}(x)+\sum_{i=0}^{n} \phi_{i}(x) \nabla \widetilde{u}_{\varepsilon}^{i}(x) \\
& =\sum_{i=0}^{n} \nabla \phi_{i}(x) \int_{B\left(x_{i}^{\varepsilon}, \varepsilon\right)} \rho_{\varepsilon}\left(x_{i}^{\varepsilon}-y\right) u(y) \mathrm{d} y+\sum_{i=0}^{n} \phi_{i}(x) \int_{B\left(x_{i}^{\varepsilon}, \varepsilon\right)} \rho_{\varepsilon}\left(x_{i}^{\varepsilon}-y\right) \nabla u(y) \mathrm{d} y .
\end{aligned}
$$

- If $x \in \omega$, then $B\left(x_{i}^{\varepsilon}, \varepsilon\right) \subset \omega$ and $\nabla u(y)=0$ a.e. $y$ in $\omega$ (since $u$ is a constant on $\omega$ ), we have $\nabla u_{\varepsilon}(x)=0$ and therefore $F^{*}\left(x, \nabla u_{\varepsilon}(x)\right)=0 \leq 1$.

- If $x \in \bar{\Omega} \backslash \omega$, there exists a sequence of small $\varepsilon$ (depending on $x$ ) such that $x_{i}^{\varepsilon} \in \Omega \backslash \omega$ for all $i=0, \ldots, n$. We have that

$$
\begin{aligned}
F^{*}\left(x, \nabla u_{\varepsilon}(x)\right) \leq & F^{*}\left(x, \sum_{i=0}^{n} \nabla \phi_{i}(x) \int_{B\left(x_{i}^{\varepsilon}, \varepsilon\right)} \rho_{\varepsilon}\left(x_{i}^{\varepsilon}-y\right) u(y) \mathrm{d} y\right) \\
& +\sum_{i=0}^{n} \phi_{i}(x) \int_{B\left(x_{i}^{\varepsilon}, \varepsilon\right)} F^{*}(x, \nabla u(y)) \rho_{\varepsilon}\left(x_{i}^{\varepsilon}-y\right) \mathrm{d} y \\
\leq & F^{*}\left(x, \sum_{i=0}^{n} \nabla \phi_{i}(x) \int_{B\left(x_{i}^{\varepsilon}, \varepsilon\right)} \rho_{\varepsilon}\left(x_{i}^{\varepsilon}-y\right) u(y) \mathrm{d} y\right) \\
& +1+\sum_{i=0}^{n} \phi_{i}(x) \underset{B\left(x_{i}^{\varepsilon}, \varepsilon\right)}{\left(F^{*}(x, \nabla u(y))-F^{*}(y, \nabla u(y))\right) \rho_{\varepsilon}\left(x_{i}^{\varepsilon}-y\right) \mathrm{d} y .}
\end{aligned}
$$

Since $F^{*}(x, q)$ is finite and continuous on $(\bar{\Omega} \backslash \omega) \times \mathbb{R}^{N}$, we have $F^{*}(x, \nabla u(y))-$ $F^{*}(y, \nabla u(y)) \rightarrow 0$ as $y \rightarrow x$ whenever $\nabla u(y)$ exists.

Letting $\varepsilon \rightarrow 0$, we obtain $\limsup _{\varepsilon \rightarrow 0} F^{*}\left(x, \nabla u_{\varepsilon}(x)\right) \leq 1$.

Step 4 (proof of (ii)). Set

$$
u_{\varepsilon}:=\frac{1}{1+C \varepsilon+w(\varepsilon)} \sum_{i=0}^{n} \phi_{i} \widetilde{u}_{\varepsilon}^{i},
$$


where the constant $C$ is chosen later and

$$
w(\varepsilon):=\sup _{x, y}\left\{\left|F^{*}(x, q)-F^{*}(y, q)\right|: x, y \in \bar{\Omega} \backslash \omega,|x-y| \leq M \varepsilon,|q| \leq\|\nabla u\|_{L^{\infty}}\right\},
$$

with a fixed constant $M \geq \max _{1 \leq i \leq n}\left\{\lambda_{z_{i}}+1\right\}, \lambda_{z_{i}}$ is given in Step 1. We show that $u_{\varepsilon}$ satisfies all the desired properties. By the assumption of (ii), $w(\varepsilon) \rightarrow 0$ as $\varepsilon \rightarrow 0$. It is clear that $u_{\varepsilon} \in C_{c}^{\infty}\left(\mathbb{R}^{N}\right)$ and

$$
u_{\varepsilon} \rightrightarrows \sum_{i=0}^{n} \phi_{i} u=u \text { on } \bar{\Omega}
$$

At last, we show that $F^{*}\left(x, \nabla u_{\varepsilon}(x)\right) \leq 1 \forall x \in \bar{\Omega}$. Similar to Step 3, we have $F^{*}\left(x, \nabla u_{\varepsilon}(x)\right)=$ $0 \leq 1$ for all $x \in \omega$. Let us now prove for the case $x \in \bar{\Omega} \backslash \omega$. Using the fact that

$$
\sum_{i=0}^{n} \nabla \phi_{i}(x) u(x)=0 \text { for all } x \in \bar{\Omega},
$$

we have

$$
\sum_{i=0}^{n} \nabla \phi_{i}(x) \int_{B\left(x_{i}^{\varepsilon}, \varepsilon\right)} \rho_{\varepsilon}\left(x_{i}^{\varepsilon}-y\right) u(y) \mathrm{d} y=\sum_{i=0}^{n} \nabla \phi_{i}(x)\left(\int_{B\left(x_{i}^{\varepsilon}, \varepsilon\right)} \rho_{\varepsilon}\left(x_{i}^{\varepsilon}-y\right) u(y) \mathrm{d} y-u(x)\right) .
$$

Moreover,

$$
\begin{aligned}
\int_{B\left(x_{i}^{\varepsilon}, \varepsilon\right)} \rho_{\varepsilon}\left(x_{i}^{\varepsilon}-y\right) u(u) \mathrm{d} y-u(x) \mid & \leq\left|\int_{B\left(x_{i}^{\varepsilon}, \varepsilon\right)} \rho_{\varepsilon}\left(x_{i}^{\varepsilon}-y\right)\left(u(y)-u\left(x_{i}^{\varepsilon}\right)\right) \mathrm{d} y\right|+\left|u\left(x_{i}^{\varepsilon}\right)-u(x)\right| \\
& \leq C_{1} \varepsilon \forall i=0, \ldots, n,
\end{aligned}
$$

where the constant $C_{1}$ depends only on $\operatorname{Lip}\left(\gamma_{z_{i}}\right)$ and the Lipschitz constant of $u$ on $\bar{\Omega}$. Thus, by combining this with (3.6),

$$
\left|\sum_{i=0}^{n} \nabla \phi_{i}(x) \int_{B\left(x_{i}^{\varepsilon}, \varepsilon\right)} \rho_{\varepsilon}\left(x_{i}^{\varepsilon}-y\right) u(y) \mathrm{d} y\right| \leq C_{2} \varepsilon \forall x \in \Omega,
$$

where $C_{2}$ depends only on $C_{1}$ and $\left\|\nabla \phi_{i}\right\|_{L^{\infty}}$.

Since $F^{*}(x, q)$ is bounded from above on $(\bar{\Omega} \backslash \omega) \times\{|q| \leq 1\}$, there exists $K>0$ such that $F^{*}(x, q) \leq K|q|$ for all $x \in \bar{\Omega} \backslash \omega, q \in \mathbb{R}^{N}$ and therefore

$$
F^{*}\left(x, \sum_{i=0}^{n} \nabla \phi_{i}(x) \int_{B\left(x_{i}^{\varepsilon}, \varepsilon\right)} \rho_{\varepsilon}\left(x_{i}^{\varepsilon}-y\right) u(y) \mathrm{d} y\right) \leq C_{3} \varepsilon \text { for all } x \in \bar{\Omega} \backslash \omega .
$$


Now, if $y \in B\left(x_{i}^{\varepsilon}, \varepsilon\right)$ then $|x-y| \leq\left|x-x_{i}^{\varepsilon}\right|+\left|x_{i}^{\varepsilon}-y\right| \leq M \varepsilon$. So we obtain, in view of (3.5), $F^{*}\left(x, \nabla u_{\varepsilon}(x)\right)$

$\leq \frac{1}{1+C \varepsilon+w(\varepsilon)}\left[C_{3} \varepsilon+1+\sum_{i=0}^{n} \phi_{i}(x) \int_{B\left(x_{i}^{\varepsilon}, \varepsilon\right)} \rho_{\varepsilon}\left(x_{i}^{\varepsilon}-y\right)\left(F^{*}(x, \nabla u(y))-F^{*}(y, \nabla u(y))\right) \mathrm{d} y\right]$

$\leq \frac{C_{3} \varepsilon+1+w(\varepsilon)}{1+C \varepsilon+w(\varepsilon)}$

$\leq 1$ (choose a constant $\left.C \geq C_{3}\right)$.

Here, we have used $\nabla u(y)=0$ in $\omega$ and the boundary $\partial \omega$ is negligble. Thus,

$$
\begin{aligned}
& \int_{B\left(x_{i}^{\varepsilon}, \varepsilon\right)} \rho_{\varepsilon}\left(x_{i}^{\varepsilon}-y\right)\left(F^{*}(x, \nabla u(y))-F^{*}(y, \nabla u(y))\right) \mathrm{d} y \\
= & \int_{B\left(x_{i}^{\varepsilon}, \varepsilon\right) \cap(\bar{\Omega} \backslash \omega)} \rho_{\varepsilon}\left(x_{i}^{\varepsilon}-y\right)\left(F^{*}(x, \nabla u(y))-F^{*}(y, \nabla u(y))\right) \mathrm{d} y \leq w(\varepsilon) .
\end{aligned}
$$

\section{Monge-Kantorovich equation}

In this section, we present a generalization of Monge-Kantorovich equation for degenerate Finsler metrics:

$$
\begin{cases}-\operatorname{div} \Phi=f_{1}-f_{0} & \text { in } \mathcal{D}^{\prime}(\bar{\Omega}) \\ \frac{\Phi}{|\Phi|}(x) \cdot D_{|\Phi|} u(x)=F\left(x, \frac{\Phi}{|\Phi|}(x)\right) & |\Phi| \text {-a.e. } \\ F^{*}(x, \nabla u(x)) \leq 1 & \text { a.e. in } \Omega,\end{cases}
$$

where $D_{|\Phi|} u$ is the tangential gradient w.r.t. the total variation measure $|\Phi|$ (see $[4,5,11]$ ). This equation turns out to be a standard Monge-Kantorovich equation if $F(x, p)=|p|$ is the Euclidean norm (independent of $x$ ). Also, it is worth noting that we do not use the constraint $F^{*}\left(x, D_{|\Phi|} u(x)\right) \leq 1$ as in [8].

Theorem 4.3. (i) For any $u$ such that $F^{*}(x, \nabla u(x)) \leq 1$ a.e. in $\Omega$ and any $\Phi \in \mathcal{M}(\bar{\Omega})^{N}$ such that $-\operatorname{div} \Phi=f_{1}-f_{0}$ in $\mathcal{D}^{\prime}(\bar{\Omega})$, then

$$
\frac{\Phi}{|\Phi|}(x) \cdot D_{|\Phi|} u(x) \leq F\left(x, \frac{\Phi}{|\Phi|}(x)\right) \quad|\Phi|-\text { a.e. } x \in \bar{\Omega} .
$$

(ii) $u$ and $\Phi$ are optimal solutions, respectively, to the Kantorovich dual problem (DP) and the minimal flow problem $(B)$ if and only if $(u, \Phi)$ satisfies the system (MKE). 
Proof. (i) Let $B$ be any Borel subset of $\bar{\Omega}$. By Lemma 3.1, there exists a sequence of smooth functions $u_{\varepsilon}$ such that $u_{\varepsilon} \rightrightarrows u$ on $\bar{\Omega}$ and $\limsup _{\varepsilon \rightarrow 0} F^{*}\left(x, \nabla u_{\varepsilon}(x)\right) \leq 1$ for all $x \in \bar{\Omega}$. We get

$$
\begin{aligned}
\int_{B} \frac{\Phi}{|\Phi|}(x) \cdot D_{|\Phi|} u(x) \mathrm{d}|\Phi|(x) & =\lim _{\varepsilon \rightarrow 0} \int_{B} \frac{\Phi}{|\Phi|}(x) \cdot \nabla u_{\varepsilon}(x) \mathrm{d}|\Phi|(x) \\
& \leq \limsup _{\varepsilon \rightarrow 0} \int_{B} F^{*}\left(x, \nabla u_{\varepsilon}(x)\right) F\left(x, \frac{\Phi}{|\Phi|}(x)\right) \mathrm{d}|\Phi|(x) \\
& \leq \int_{B} F\left(x, \frac{\Phi}{|\Phi|}(x)\right) \mathrm{d}|\Phi|(x),
\end{aligned}
$$

where the last inequality follows from Fatou's lemma and $\limsup _{\varepsilon \rightarrow 0} F^{*}\left(x, \nabla u_{\varepsilon}(x)\right) \leq 1$ for all $x$ in $\bar{\Omega}$. By the arbitrariness of Borel set $B$, it follows that

$$
\frac{\Phi}{|\Phi|}(x) \cdot D_{|\Phi|} u(x) \leq F\left(x, \frac{\Phi}{|\Phi|}(x)\right) \text { for all }|\Phi| \text {-a.e. } x \text { in } \bar{\Omega} .
$$

(ii) Let $u$ and $\Phi$ be optimal solutions to the Kantorovich dual problem and minimal flow problem, that is, $F^{*}(x, \nabla u(x)) \leq 1$ a.e. $x \in \Omega$ and $\Phi \in \mathcal{M}(\bar{\Omega})^{N},-\operatorname{div} \Phi=f_{1}-f_{0}$ in $\mathcal{D}^{\prime}(\bar{\Omega})$, and by the duality in Subsection 2.2 ,

$$
\int_{\bar{\Omega}} u \mathrm{~d}\left(f_{1}-f_{0}\right)=\int_{\bar{\Omega}} F\left(x, \frac{\Phi}{|\Phi|}(x)\right) \mathrm{d}|\Phi|(x) .
$$

On the other hand, taking $u$ as a test function in the divergence constraint, we get

$$
\int_{\bar{\Omega}} u \mathrm{~d}\left(f_{1}-f_{0}\right)=\int_{\bar{\Omega}} \frac{\Phi}{|\Phi|}(x) \cdot D_{|\Phi|} u(x) \mathrm{d}|\Phi|(x)
$$

and therefore

$$
\int_{\bar{\Omega}} \frac{\Phi}{|\Phi|}(x) \cdot D_{|\Phi|} u(x) \mathrm{d}|\Phi|(x)=\int_{\bar{\Omega}} F\left(x, \frac{\Phi}{|\Phi|}(x)\right) \mathrm{d}|\Phi|(x) .
$$

By (i) above, we deduce that

$$
\frac{\Phi}{|\Phi|}(x) \cdot D_{|\Phi|} u(x)=F\left(x, \frac{\Phi}{|\Phi|}(x)\right) \quad|\Phi| \text {-a.e. } x \text { in } \bar{\Omega} .
$$

Conversely, if $(u, \Phi)$ satisfies the system (MKE), then

$$
\int_{\bar{\Omega}} u \mathrm{~d}\left(f_{1}-f_{0}\right)=\int_{\bar{\Omega}} \frac{\Phi}{|\Phi|}(x) \cdot D_{|\Phi|} u(x) \mathrm{d}|\Phi|(x)=\int_{\bar{\Omega}} F\left(x, \frac{\Phi}{|\Phi|}(x)\right) \mathrm{d}|\Phi|(x),
$$

which implies the optimalities of $u$ and $\Phi$, in view of the duality.

Acknowledgements. The research is funded by Vietnam National Foundation for Science and Technology Development (NAFOSTED) under grant number 101.01- 2018.309. 


\section{References}

[1] M. Beckmann. A continuous model of transportation. Econometrica: Journal of the Econometric Society, 20(4):643-660, 1952.

[2] J.-D. Benamou, G. Carlier, and R. Hatchi. A numerical solution to Monge's problem with a Finsler distance as cost. ESAIM: Mathematical Modelling and Numerical Analysis, 52(6):2133-2148, 2018.

[3] G. Bouchitté and G. Buttazzo. Characterization of optimal shapes and masses through Monge-Kantorovich equation. Journal of the European Mathematical Society, 3(2):139-168, 2001.

[4] G. Bouchitte, G. Buttazzo, and P. Seppecher. Energies with respect to a measure and applications to low dimensional structures. Calculus of Variations and Partial Differential Equations, 5(1):37-54, 1997.

[5] G. Bouchitté, G. Buttazzo, and P. Seppecher. Shape optimization solutions via Monge-Kantorovich equation. Comptes Rendus de l'Académie des Sciences-Series I-Mathematics, 324(10):1185-1191, 1997.

[6] H. Brezis. Functional analysis, Sobolev spaces and partial differential equations. Springer Science \& Business Media, 2010.

[7] L. C. Evans and W. Gangbo. Differential equations methods for the MongeKantorovich mass transfer problem. Memoirs of the American Mathematical Society, 137:1-66, 1999.

[8] N. Igbida, J. M. Mazón, J. D. Rossi, and J. Toledo. Optimal mass transportation for costs given by Finsler distances via p-Laplacian approximations. Advances in Calculus of Variations, 11(1):1-28, 2018.

[9] N. Igbida and V. T. Nguyen. Augmented Lagrangian method for optimal partial transportation. IMA Journal of Numerical Analysis, 38(1):156-183, 2018.

[10] N. Igbida and V. T. Nguyen. Optimal partial mass transportation and obstacle Monge-Kantorovich equation. Journal of Differential Equations, 264(10):6380-6417, 2018.

[11] C. Jimenez. Dynamic formulation of optimal transport problems. Journal of Convex Analysis, 15(3):593-622, 2008.

[12] F. Santambrogio. Optimal transport for applied mathematicians, volume 87. Birkäuser, 2015. 
[13] C. Villani. Topics in optimal transportation, volume 58. American Mathematical Society, 2003.

[14] C. Villani. Optimal transport: old and new, volume 338. Springer Science \& Business Media, 2008. 